\title{
High-resolution $\mathrm{CO}$ observations towards the bright eastern knot of the SNR Puppis A
}

\author{
S. Paron ${ }^{1, \star}$, G. Dubner ${ }^{1, \star \star, \star \star \star}$, E. Reynoso ${ }^{1,2, \star \star}$, and M. Rubio ${ }^{3}$ \\ 1 Instituto de Astronomía y Física del Espacio (IAFE), CC 67, Suc. 28, 1428 Buenos Aires, Argentina \\ e-mail: sparon@iafe.uba.ar \\ 2 Departamento de Física, Facultad de Ciencias Exactas y Naturales, UBA, Buenos Aires, Argentina \\ 3 Departamento de Astronomía, Universidad de Chile, Casilla 36-D, Santiago, Chile
}

Received 8 June 2007 / Accepted 3 January 2008

\section{ABSTRACT}

\begin{abstract}
Aims. This paper reports molecular observations towards the bright eastern knot (BEK) in the SNR Puppis A, a feature where radio and $\mathrm{X}$-ray studies suggest that the shock front is interacting with a dense molecular clump.

Methods. We performed high-resolution millimetric observations towards the BEK of Puppis A using the SEST telescope in the ${ }^{12} \mathrm{CO} J=1-0$ and $2-1$ lines (beams of $45^{\prime \prime}$ and $23^{\prime \prime}$, respectively). More extended, lower angular resolution ${ }^{12} \mathrm{CO} J=1-0$ observations taken from NANTEN archival data were also analyzed to obtain a complete picture.

Results. In the velocity range near $16 \mathrm{~km} \mathrm{~s}^{-1}$, which is the Puppis A systemic velocity, our study revealed two important properties: (i) no dense molecular gas is detected immediately adjacent to the eastern border of the BEK and (ii) the molecular clump detected very close to the radiocontinuum maximum is probably located in the foreground along the line of sight and has not yet been reached by the SNR shock front. We propose two possible scenarios for explaining the absence of molecular emission eastwards of the BEK border of Puppis A. Either the shock front has completely engulfed and destroyed a molecular clump or the shock front is interacting with part of a larger cloud, and we do not detect $\mathrm{CO}$ emission immediately beyond it because the molecules have been dissociated by photodissociation and by reactions with photoionized material due to the radiative precursor.
\end{abstract}

Key words. ISM: molecules - ISM: clouds - ISM: supernova remnants

\section{Introduction}

Shock waves generated by supernova remnants (SNRs) can accelerate, compress, heat, fragment or even destroy surrounding interstellar clouds. Strong shock-cloud interactions can enhance or reduce abundances of different molecular species with respect to quiescent cloud conditions. Observations of molecular gas associated with SNRs provide information essential to understand the physics and chemistry involved in these processes.

Puppis A is a Galactic SNR that has been extensively studied in the whole electromagnetic spectrum. In radio continuum, Puppis A appears as an asymmetric clumpy shell with the brightest section along the eastern border (Fig. 1a) (Castelletti et al. 2006 and references therein) presenting a good correlation with soft X-ray emission (Petre et al. 1982; Aschenbach 1988). The $\mathrm{X}$-ray emission includes both extended features and compact knots, the most conspicuous of which is the bright eastern knot (BEK; Petre et al. 1982) that coincides with an indentation in the shock front of the SNR as seen in radio. Such morphology suggests an interaction between the SNR and a dense interstellar clump. Hwang et al. (2005) presented ACIS Chandra X-ray images and spectral data of the region around the BEK. They conclude that a cloud-shock interaction in a relatively late phase of evolution is taking place near the BEK, while closer to the

^ Post-Doctoral Fellow of CONICET, Argentina.

$\star \star$ Member of the Carrera del Investigador Científico of CONICET, Argentina.

$\star \star \star$ Visiting Astronomer, European Southern Observatory forward shock in the BEK region, the SNR has recently interacted with a more dense and extended obstacle.

The CO studies of the interstellar medium surrounding Puppis A performed by Dubner \& Arnal (1988) with angular resolution of 8.7, revealed the existence of a chain of molecular clouds concentrated along the E and NE periphery of the remnant. Studies in infrared wavelengths of Puppis A also suggest the existence of interstellar clouds along the eastern border of the SNR (Arendt et al. 1991). From the atomic and molecular studies (Dubner \& Arnal 1988; Reynoso et al. 1995), a systemic velocity of $v_{L S R} \sim+16 \mathrm{~km} \mathrm{~s}^{-1}$ and a kinematical distance of $2.2 \pm 0.3 \mathrm{kpc}$ were derived for Puppis A, a distance later confirmed by Reynoso et al. (2003) based on interferometric, high resolution HI data in the direction of the associated radio quiet neutron star RX J0822-4300 located within the remnant.

In this work, we present high resolution SEST observations performed in the ${ }^{12} \mathrm{CO} J=1-0$ and $J=2-1$ lines towards the BEK region, complemented with lower resolution molecular mapping of a larger region including the BEK based on archival data of the NANTEN telescope. The posibility of interaction between the SNR shock front and the surrounding gas is locally investigated in an attempt to understand the origin of the enhancement observed both in X-ray and radio continuum emissions.

\section{Observations}

The high-resolution ${ }^{12} \mathrm{CO}$ data were acquired during March 9 to 11, 2000 with the $15 \mathrm{~m}$ Swedish-ESO Submillimetre Telescope (SEST) that operated in La Silla (Chile). The angular resolutions 

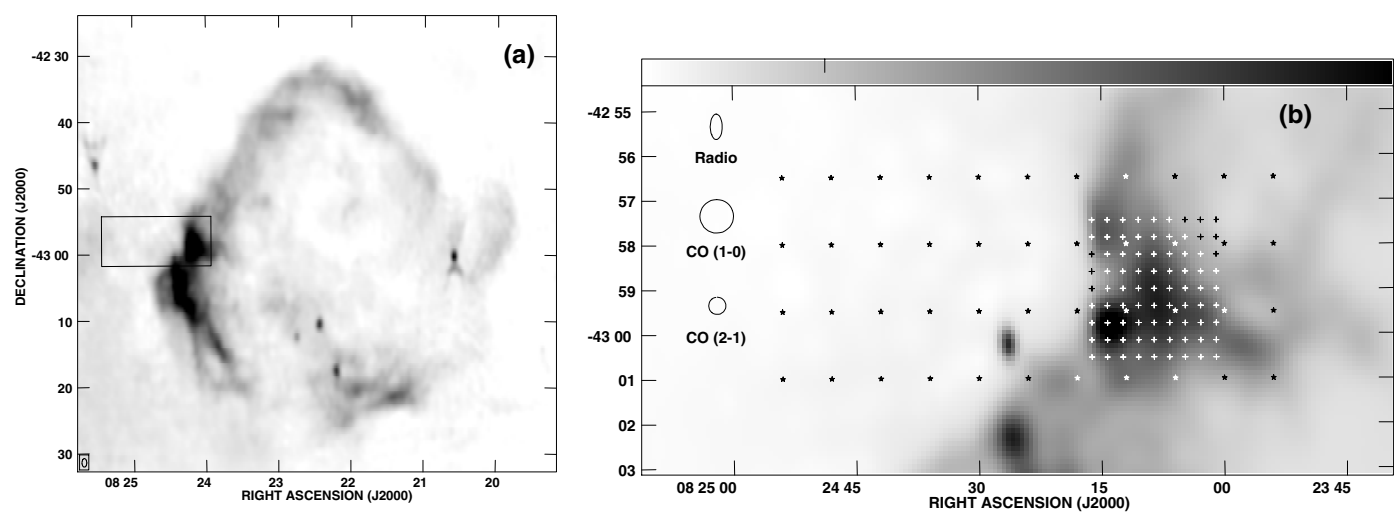

Fig. 1. a) Radio continuum image of Puppis A at $1425 \mathrm{MHz}$ (from Castelletti et al. 2006). The rectangle shows the region surveyed with SEST in the ${ }^{12} \mathrm{CO} J=1-0$ and $J=2-1$ lines. b) Observed region in detail. The pointings are indicated with crosses and stars. The respective beams are shown to the left.

of this telescope were $45^{\prime \prime}$ and $23^{\prime \prime}$ for the ${ }^{12} \mathrm{CO} J=1-0$ and $J=2-1$ transitions respectively. An Acousto-Optical spectrometer was used as back end, consisting of a narrow band highresolution (HRS) spectrometer with 1000 channels, bandwidth $80 \mathrm{MHz}$, and channel separation $41.7 \mathrm{kHz}$ (corresponding to $0.108 \mathrm{~km} \mathrm{~s}^{-1}$ for the ${ }^{12} \mathrm{CO} J=1-0$ and $0.054 \mathrm{~km} \mathrm{~s}^{-1}$ for the $\left.{ }^{12} \mathrm{CO} J=2-1\right)$. The observed velocity ranges were $\left[-40 \mathrm{~km} \mathrm{~s}^{-1}\right.$, $\left.+60 \mathrm{~km} \mathrm{~s}^{-1}\right]$ and $\left[-10 \mathrm{~km} \mathrm{~s}^{-1},+40 \mathrm{~km} \mathrm{~s}^{-1}\right]$ for the ${ }^{12} \mathrm{CO} J=1-0$ and $J=2-1$ transitions respectively.

Figure 1a displays the SNR Puppis A as observed in radio continuum at $1420 \mathrm{MHz}$. The rectangle shows the region surveyed with the SEST telescope. In this region the ${ }^{12} \mathrm{CO} J=1-0$ and $2-1$ transitions were observed in 81 pointings with a grid spacing of $23^{\prime \prime}$. The pointings are shown as crosses in the enlargement included in Fig. 1b. This region covers a square of approximately $3.35 \times 3.35$ centered at $\mathrm{RA}=8^{\mathrm{h}} 24^{\mathrm{m}} 8.5^{\mathrm{s}}, \mathrm{Dec}=-42^{\circ} 59^{\prime} 00^{\prime \prime}$ (J2000). The whole field was observed twice with an integration time of $105 \mathrm{~s}$ per position each time. Beyond this square, 44 additional pointings, also shown in Fig. $1 \mathrm{~b}$ as stars, were observed in both CO lines with a grid spacing of $90^{\prime \prime}$, except for one pointing (at $\mathrm{RA}=8^{\mathrm{h}} 23^{\mathrm{m}} 54^{\mathrm{s}}$ and $\mathrm{Dec}=-43^{\circ} 01^{\prime} 00^{\prime \prime}$ ) that was observed only at the ${ }^{12} \mathrm{CO} J=1-0$ transition.

All spectra were Hanning smoothed to improve the signal-tonoise ratio. The rms noises in main-beam brightness temperature are $\sigma_{1-0} \sim 0.15 \mathrm{~K}$ and $\sigma_{2-1} \sim 0.30 \mathrm{~K}$ for the 81 pointings observed over the BEK and $\sigma_{1-0} \sim 0.20 \mathrm{~K}$ and $\sigma_{2-1} \sim 0.15 \mathrm{~K}$ for the 44 additional pointings for the ${ }^{12} \mathrm{CO} J=1-0$ and $2-1$ transitions respectively. The spectra were processed using the XSpec software package developed at the Onsala Space Observatory. Images were produced using the AIPS package.

Additionally we used ${ }^{12} \mathrm{CO} J=1-0$ data acquired with the $4 \mathrm{~m}$ radiotelescope NANTEN that operated in Las Campanas Observatory (Chile), to explore a more extended region along the eastern border of Puppis A. These data are part of the study in the direction of the Gum Nebula carried out by Yamaguchi et al. (1999). The angular resolution of the telescope is 2:6 and the grid sampling is $8^{\prime}$ in 1 and $b$. The total bandwidth and the effective spectral resolution were $40 \mathrm{MHz}$ and $35 \mathrm{kHz}$ respectively, corresponding to a velocity coverage of $100 \mathrm{~km} \mathrm{~s}^{-1}$ and a velocity resolution of $\sim 0.1 \mathrm{~km} \mathrm{~s}^{-1}$. At this velocity resolution the typical rms noise was $\sim 0.7 \mathrm{~K}$ (Yamaguchi et al. 1999).

\section{Results}

Figure 2 shows ${ }^{12} \mathrm{CO} J=1-0$ and $J=2-1$ spectra in the whole observed velocity range obtained from the average of

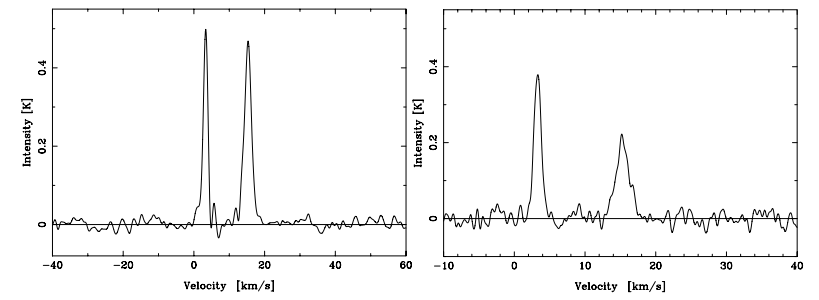

Fig. 2. ${ }^{12} \mathrm{CO} J=1-0$ (left) and $J=2-1$ (right) average profiles from the 81 observed spectra towards the BEK of Puppis A (crosses in Fig. 1b).

the 81 pointings towards the BEK of Puppis A. Two velocity components are present, near 3 and $16 \mathrm{~km} \mathrm{~s}^{-1}$. The narrow $3 \mathrm{~km} \mathrm{~s}^{-1}$ component probably originates in foreground unperturbed gas crossed by the line of sight, likely to be associated with the Gum Nebula (Reynoso \& Dubner 1997; Yamaguchi et al. 1999). This component will therefore not be further considered.

To analyze the surroundings of the BEK area we first inspect the distribution of the molecular gas near $v \sim 16 \mathrm{~km} \mathrm{~s}^{-1}$ in an extended region $\left(1^{\circ} \times 1^{\circ}\right)$ around Puppis $\mathrm{A}$ based on the coarser sampled NANTEN observations. In Fig. 3 we present the ${ }^{12} \mathrm{CO} J=1-0$ emission integrated each $0.5 \mathrm{~km} \mathrm{~s}^{-1}$ in the range between 13.5 and $17.5 \mathrm{~km} \mathrm{~s}^{-1}$. The rms noise, calculated from regions free of emission, is $0.06 \mathrm{~K} \mathrm{~km} \mathrm{~s}^{-1}$. The NANTEN data confirm the presence of molecular gas eastwards of the SNR around $v \sim 16 \mathrm{~km} \mathrm{~s}^{-1}$ as suggested before based on lower angular resolution observations (Dubner \& Arnal 1988). The maximum of the molecular complex, however, is shifted to the east in at least half a degree from the border of the SNR. Therefore, it is likely that the SN shock expanding towards the east encountered moderately dense molecular gas, maybe with some embedded denser clumps that cannot be resolved with these observations. The morphological matching between the edges of the SNR and the molecular complex between 13.5 and $15.0 \mathrm{~km} \mathrm{~s}^{-1}$ is striking, but the ${ }^{12} \mathrm{CO} J=1-0$ emission observed between $v=15.5$ and $16.5 \mathrm{~km} \mathrm{~s}^{-1}$ is clearly detached from the SNR's eastern limb. At $v=16.0 \mathrm{~km} \mathrm{~s}^{-1}$, in coincidence with the systemic velocity derived for Puppis A, a very faint molecular feature can be seen exactly covering the region of the BEK. In summary from the analysis of these moderate resolution data, in spite of the proximity of the eastern cloud to the SNR limb, it is not obvious that the SN shock is abutting upon a dense molecular cloud. In what follows, we investigate the BEK region with greater detail using the SEST data. 


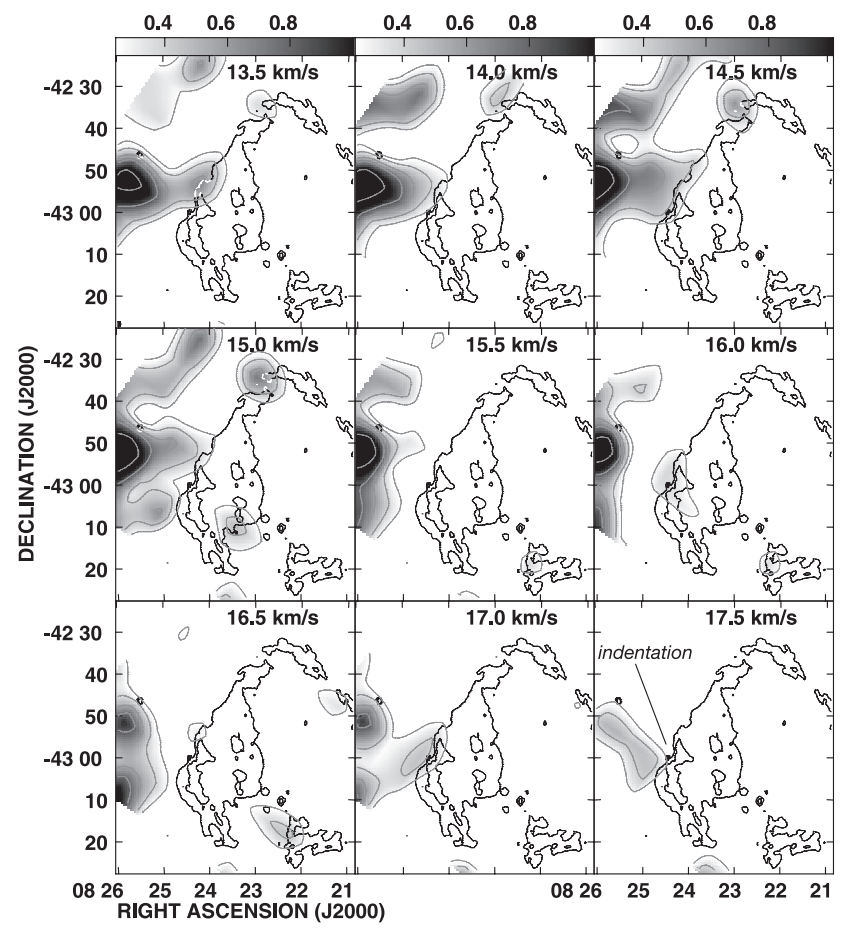

Fig. 3. ${ }^{12} \mathrm{CO} J=1-0$ emission obtained with NANTEN (from Yamaguchi et al. 1999) integrated each $0.5 \mathrm{~km} \mathrm{~s}^{-1}$. Some contours of the radio continuum emission of Puppis $\mathrm{A}$ are included. In the last frame, the position of the BEK indentation is indicated. The gray scale, in $\mathrm{K} \mathrm{km} \mathrm{s}^{-1}$, is shown at the top. The contour levels of the ${ }^{12} \mathrm{CO}$ emission are $0.25,0.40,0.60,0.80$ and $1.20 \mathrm{~K} \mathrm{~km} \mathrm{~s}^{-1}$. The rms noise of each image is $\sim 0.06 \mathrm{~K} \mathrm{~km} \mathrm{~s}^{-1}$ and the beam is 2 . 6 .
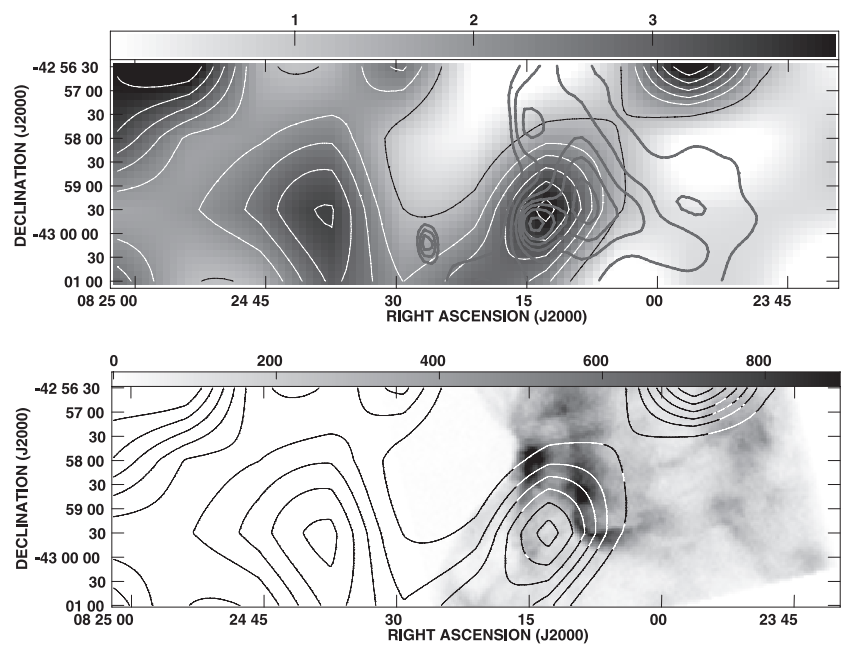

Fig. 4. Top panel: ${ }^{12} \mathrm{CO} J=1-0$ emission, integrated between 13.5 and $17.5 \mathrm{~km} \mathrm{~s}^{-1}$ obtained from the 44 pointings observed with a grid spacing of $90^{\prime \prime}$, is presented in grays and contours with levels 1.2, 2, 2.5, $3,3.5,4 \mathrm{~K} \mathrm{~km} \mathrm{~s}^{-1}$. The thick, dark grey contours correspond to the radio continuum emission of Puppis A with levels 23, 36, 50, 60, 68, 76, $87 \mathrm{mJy}$ beam $^{-1}$. Bottom panel: the same ${ }^{12} \mathrm{CO} J=1-0$ contours presented in the top panel, superimposed over the Chandra X-ray emission (image taken from the Chandra SNR Catalog).

Figure 4 displays the ${ }^{12} \mathrm{CO} J=1-0$ integrated between 13.5 and $17.5 \mathrm{~km} \mathrm{~s}^{-1}$ obtained from the SEST 44 pointings performed with a grid spacing of $90^{\prime \prime}$ (see Fig. 1) compared with the radio continuum emission (top) and with the Chandra X-ray image (bottom). A molecular clump very close to the radio continuum maximum and south of the brightest X-ray features, is detected.
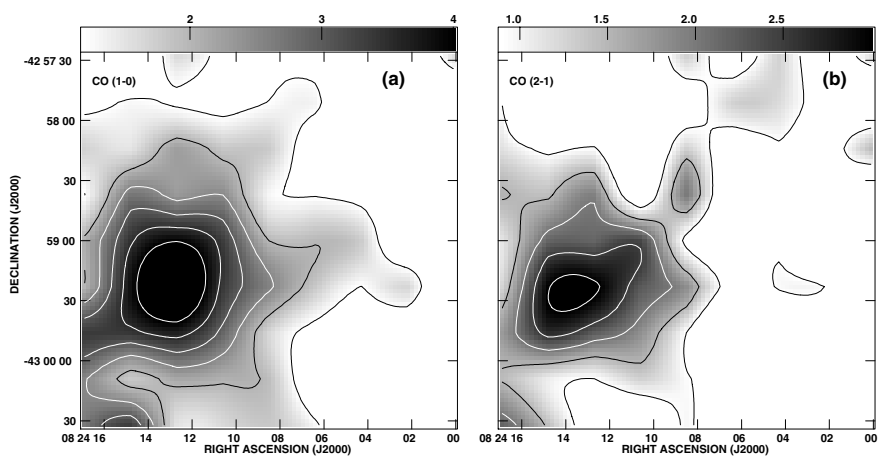

Fig. 5. ${ }^{12} \mathrm{CO}$ emission obtained from the 81 pointings observed towards the BEK. a) ${ }^{12} \mathrm{CO} J=1-0$ emission integrated between 13.5 and $17.5 \mathrm{~km} \mathrm{~s}^{-1}$. The contour levels are 1.2, 2, 2.5, 3, 3.5 and $4 \mathrm{~K} \mathrm{~km} \mathrm{~s}^{-1}$. The beam size is $45^{\prime \prime}$ and the noise level is $\sigma \sim 0.4 \mathrm{~K} \mathrm{~km} \mathrm{~s}^{-1}$. b) ${ }^{12} \mathrm{CO}$ $J=2-1$ emission integrated between 13.5 and $17.5 \mathrm{~km} \mathrm{~s}^{-1}$. The contour levels are $0.9,1.5,2,2.5$ and $3 \mathrm{~K} \mathrm{~km} \mathrm{~s}^{-1}$. The beam size is $23^{\prime \prime}$ and the noise level is $\sigma \sim 0.3 \mathrm{~K} \mathrm{~km} \mathrm{~s}^{-1}$. In both images the gray scale is shown at the top.

Clearly detached from this CO emission, there is a second concentration towards the east, probably part of the complex shown in Fig. 3, and another feature, not completely mapped, towards the northwest of the mapped region, well inside the SNR shell. These observations confirm that no high density $\mathrm{CO}$ gas is observed immediately adjacent to the eastern border in this part of Puppis A in spite of the radiocontinuum and X-ray emission morphologies, suggestive of higher compression in this region due to the shock front encountering a dense molecular cloud.

To analyze the molecular clump observed very close to the radicontinuum maximum, in Fig. 5 we display the ${ }^{12} \mathrm{CO} J=$ $1-0$ and $J=2-1$ distribution integrated between 13.5 and $17.5 \mathrm{~km} \mathrm{~s}^{-1}$, obtained from the 81 pointings performed with the best grid spacing. This figure shows with more detail the molecular clump described above. Taking into account that the ${ }^{12} \mathrm{CO} J=2-1$ line is optically thinner and surveys denser gas than the $J=1-0$ line (e.g. Sakamoto et al. 1994), we will use it to compare with the radio continuum and X-ray emissions. In Fig. 6 , the same ${ }^{12} \mathrm{CO} J=2-1$ contours shown in Fig. $5 \mathrm{~b}$ are displayed over the radio continuum emission (Fig. 6a), which appears in greys and with black contours, and over the Chandra X-ray emission (Fig. 6b). These images show that the maximum of the molecular clump is located spatially very close to, but not exactly coincident with the maximum of the radio continuum. At the same time the clump is clearly anticorrelated with the $\mathrm{X}$-ray emission. There is, however, some molecular/X-ray coincidence: the western border of the clump appears superimposed onto the arched bright X-ray feature called "the bar" by Hwang et al. (2005).

In what follows we calculate the physical parameters of the only molecular clump spatially and kinematically coincident with the SNR bright eastern radio knot. If we consider the limit of the ${ }^{12} \mathrm{CO} J=1-0$ structure observed in Fig. 5a as the $3.5 \mathrm{~K} \mathrm{~km} \mathrm{~s}^{-1}$ contour ( $\sim 8$ times above the noise level), a well delimited region of $\sim 1^{\prime}$ diameter is defined. Assuming a distance of $2.2 \mathrm{kpc}$, the linear diameter of this region is $0.3 \mathrm{pc}$. Using the ${ }^{12} \mathrm{CO} J=1-0$ integrated emission and the conversion factor $X=1.9 \times 10^{20} \mathrm{~cm}^{-2}\left(\mathrm{~K} \mathrm{~km} \mathrm{~s}^{-1}\right)^{-1}$ (Bloemen et al. 1986) we can estimate the molecular mass of this feature to be $M \simeq 9.5 M_{\odot}$ and its volumetric density, $n \simeq 1.7 \times 10^{4} \mathrm{~cm}^{-3}$. 

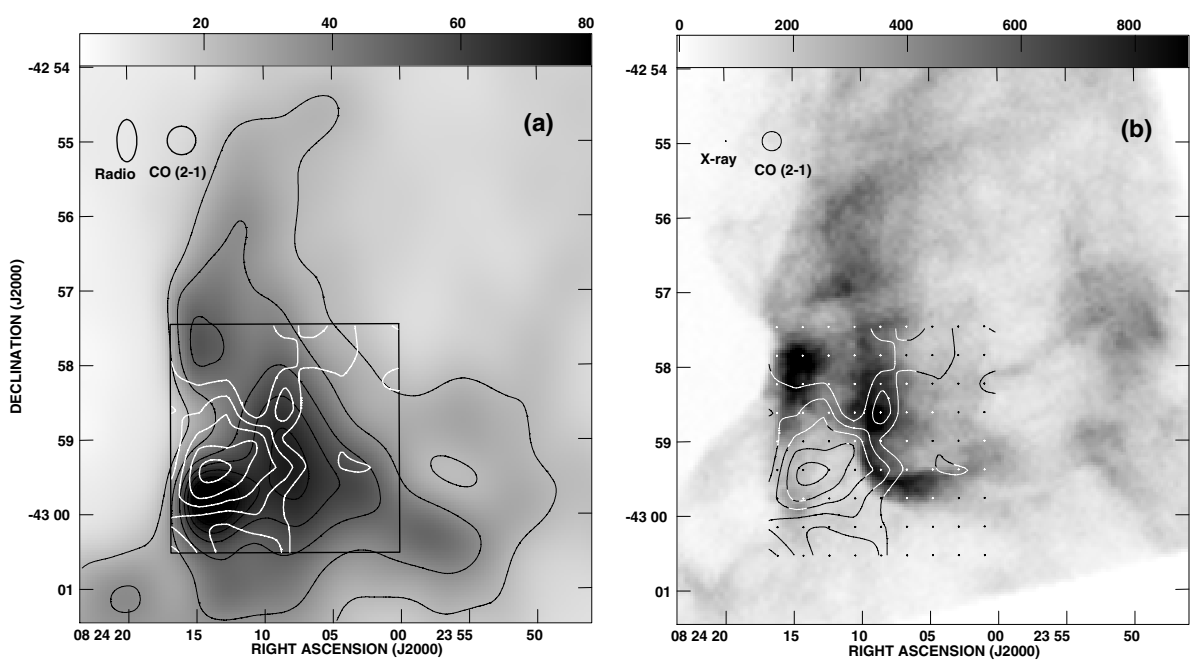

Fig. 6. a) ${ }^{12} \mathrm{CO} J=2-1$ contours over the radio continuum emission of Puppis A, the levels are the same of Fig. 5b. Also some contours (in black) of the radio continuum are included with levels $23,36,50,60,68,76,87 \mathrm{mJy}_{\text {beam }}^{-1}$. The gray scale, in mJy beam ${ }^{-1}$, is shown at the top. b) Same ${ }^{12} \mathrm{CO} J=2-1$ contours over the Chandra X-ray emission (from the Chandra SNR Catalog). The points indicate the pointings for the molecular observations. The respective beams are shown at the top left corner of each image.

\section{Discussion}

Hwang et al. (2005) analyzed in detail the X-ray emission morphology near the region of the BEK of Puppis A, reporting the detection of two different morphological components in the area. In particular these authors point out that the indentation immediately east of the X-ray emission peak strongly suggests that the shock front has recently interacted with a dense obstacle and is wrapping it around. They conclude that this obstacle is probably quite extended along the line of sight and projection effects must be important. The presence of multiple clouds in the line of sight is a possibility previously noted by Blair et al. (1995). What is surprising is that the new high-resolution millimetric observations revealed that there is no molecular gas in a region immediately adjacent to the eastern border of the BEK. To explain the lack of dense molecular gas in a fringe about $\sim 3^{\prime}$ wide (about $\sim 2 \mathrm{pc}$ at $2.2 \mathrm{kpc}$ ) to the east of Puppis A, in spite of the fact that the presence of a molecular complex close to the eastern border is well proved, two possible reasons can be proposed. One of them is that the shock front has completely engulfed a molecular clump and no remains are left. In this case, the compact X-ray emission could be due to evaporation of the molecular gas as proposed by Petre et al. (1982) and Teske \& Petre (1987), and the indentation could be marking the reestablishment of the shock front following the cloud passage. A similar behavior has been observed in another SNR, the Cygnus Loop (Fesen et al. 1992), where $\mathrm{H} \alpha$ observations show an isolated emission cloud along the Cygnus Loop's eastern limb and an indented shock front beyond the cloud location. Klein et al. (1994), assuming a radiative shock, developed an hydrodynamical model for the interaction of shock waves with interstellar clouds and applied it to the shocked cloud in the eastern region of the Cygnus Loop. They found that, as a result of the interaction, the blast wave diffracts around the cloud and reestablishes ahead of it, in agreement with the observations of Fesen et al. (1992). Besides, X-ray ROSAT observations (Levenson et al. 1997, 2002) show an indentation at this region of the Cygnus Loop followed by a compact bright knot interior to the shell, similarly to what is observed in Puppis A.

An alternative to explaining the lack of molecular gas adjacent to the BEK could be that the indentation is a consequence

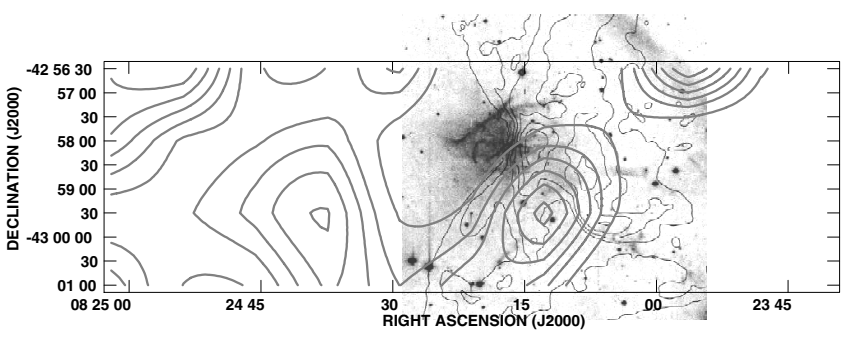

Fig. 7. [O III] emitting filaments (observed by Blair et al. 1995) with contours of the X-ray emission (image extracted from Hwang et al. 2005). The same contours of the ${ }^{12} \mathrm{CO} J=1-0$ emission presented in Fig. 4 are superimposed.

of an interaction with part of a larger cloud and we do not detect CO emission ahead of the SNR shock because of photoionization and photodissociation effects. Indeed, Blair et al. (1995) detected [O III] emitting filaments in Puppis A immediately adjacent to the BEK indentation, extending significantly beyond the eastern boundary of the X-ray emission. The authors propose that the enhanced diffuse [O III] to the east could indicate preshock photoionization while the diffuse optical emission behind the X-ray shock front may imply evaporation. Figure 7 shows the [O III] emission image of the region as taken from Blair et al. (1995) with contours of the X-ray emission (thin gray lines) and of molecular emission (thick lines) overlaid. Using the Hopkins Ultraviolet Telescope, Blair et al. (1995) reported the detection of far-ultraviolet (FUV) emission in one of the [OIII] filaments near the BEK position (at about $\mathrm{RA}=8^{\mathrm{h}} 24^{\mathrm{m}} 18.65^{\mathrm{s}}$ and Dec $=-42^{\circ} 57^{\prime} 40^{\prime}$ '50, J2000), which could confirm the existence of FUV photons capable of dissociating molecules. It is known that energetic electrons produced by X-ray ionization in molecular clouds collisionally excite the Lyman and Werner bands of $\mathrm{H}_{2}$; the subsequent radiative de-excitations generate a flux of FUV photons capable of dissociating many molecular species including the CO (Prasad \& Tarafdar 1983; Wardle 1999). It is possible that the $\mathrm{CO}$ immediately adjacent to Puppis A's shock front has been photodissociated by the FUV photons product of the X-ray ionization. Moreover, reactions between the $\mathrm{CO}$ and $\mathrm{He}^{+}$, ionized by the radiative precursor (Ghavamian et al. 2000), can dissociate the CO molecule (Prasad \& Tarafdar 1983). Far-infrared observations of the dust continuum and the 
fine structure of [CII] and [OI] at $158 \mu \mathrm{m}$ and $63 \mu \mathrm{m}$ respectively would be helpful to explore the interface between the warm photodissociated region in the cloud's surface and the cooler interior (Hollenbach \& Tielens 1999). Such detection would provide support to this last scenario.

On the other hand, between $v \sim 13.5$ and $17.5 \mathrm{~km} \mathrm{~s}^{-1}$ we observed a conspicuous molecular structure lying very close to the maximum of the radio continuum emission of the BEK. This velocity range coincides with the systemic velocity of Puppis A, $v \sim 16 \mathrm{~km} \mathrm{~s}^{-1}$, derived by Reynoso et al. (1995, 2003) and Dubner \& Arnal (1988). In order to investigate if this clump has been recently shocked, we calculated the ratio between the integrated lines $\left(R_{2-1 / 1-0}\right)$, where $R_{2-1 / 1-0}=$ $\int_{13.5}^{17.5} T_{\mathrm{CO} 2-1} \mathrm{~d} v / \int_{13.5}^{17.5} T_{\mathrm{CO} 1-0} \mathrm{~d} v$. This parameter is a useful tool to trace shocked gas. Large values in the ratio $R_{2-1 / 1-0}$ are expected to be found in shocked molecular clouds, as observed in the clouds related with the SNRs G349.7+0.2 $\left(R_{2-1 / 1-0} \sim 1.5\right.$; Dubner et al. 2004), W44 ( $\left.R_{2-1 / 1-0} \sim 1.3\right)$, IC443 $\left(R_{2-1 / 1-0} \sim\right.$ 1.3-4) and HB $21\left(R_{2-1 / 1-0} \sim 1.6-2.3\right)$ (Seta et al. 1998; Koo et al. 2001). For the observed molecular clump we obtained $R_{2-1 / 1-0} \sim 0.6$, a low value that suggests that it is quiescent gas. Figure $6 \mathrm{~b}$ shows that the molecular clump is surrounded by regions of enhanced X-ray emission. This can be an evidence that the molecular cloud is located in the foreground and absorbs the $\mathrm{X}$-ray emission from the SNR. A similar behavior is observed in a "molecular arm" towards the SNR CTB 109, where the $\mathrm{CO}$ emission is anticorrelated with the X-ray emission (Sasaki et al. 2006) and interpreted in the same way. As mentioned above, based on X-ray spectral data, Hwang et al. (2005) showed that the BEK region closer to the forward shock has lower temperatures and ionization ages and higher column densities than elsewhere in the field, concluding that these properties are signatures of a recent interaction between the SNR shock front with multiple clouds, some of which are in the remnant foreground. In this context, the discovered molecular structure must be one of these foreground clumps that has not been completely reached by the $\mathrm{SN}$ shock yet.

\section{Summary and conclusions}

The present observations enabled us to map in detail the molecular material towards the brightest X-ray emission region (BEK) in Puppis A. Our ${ }^{12} \mathrm{CO} J=1-0$ and $J=2-1$ high resolution observations show components at $v \sim 3 \mathrm{~km} \mathrm{~s}^{-1}$ and $v \sim 16 \mathrm{~km} \mathrm{~s}^{-1}$. The $v \sim 3 \mathrm{~km} \mathrm{~s}^{-1}$ component is most probably related to the Gum Nebula.

In the velocity range near $16 \mathrm{~km} \mathrm{~s}^{-1}$, our study revealed two important properties: (i) no dense molecular gas is detected immediately adjacent to the eastern border of the BEK of Puppis A (towards the SNR indentation) and (ii) the only detected cloud apparently associated with the BEK is probably located in the foreground along the line of sight and has not been reached by the $\mathrm{SN}$ shock yet.
To explain the absence of molecular emission eastwards of Puppis A in the BEK region, two scenarios were proposed. Either the shock front has completely engulfed and destroyed a molecular clump and the X-ray emission is due to the evaporation of the molecular gas, or the indentation is the consequence of an interaction with part of a larger cloud and we do not detect $\mathrm{CO}$ emission immediately beyond it because the molecules have been dissociated by photodissociation and by reactions with photoionized material due to the radiative precursor, as optical emission suggests. Far-infrared observations of the dust continuum and the fine structure of $[\mathrm{CII}]$ and $[\mathrm{OI}]$ may help to test this suggestion.

Concerning the molecular clump detected at $v \sim 16 \mathrm{~km} \mathrm{~s}^{-1}$, we estimated for it a linear size of about $0.3 \mathrm{pc}$, a molecular mass of $\sim 9.5 M_{\odot}$, and a volume density of $\sim 1.7 \times 10^{4} \mathrm{~cm}^{-3}$. The low value obtained in the ratio between the integrated lines towards this clump, $R_{2-1 / 1-0} \sim 0.6$, suggests that we are observing quiescent gas located in front of Puppis A along the line of sight.

Acknowledgements. We thank Norikazu Mizuno for kindly providing us with the NANTEN data. G.D. is very grateful to the staff of SEST for the support received during the observations, especially to the former director Dr. L.-A. Nyman. This work was supported by the CONICET grant 6433/05, UBACYT A055/04 and ANPCYT PICT 04-14018.

\section{References}

Arendt, R. G., Dwek, E., \& Petre, R. 1991, ApJ, 368, 474

Aschenbach, B. 1988, in NATO ASIC Proc. 249: Hot Thin Plasmas in Astrophysics, ed. R. Pallavicini, 185

Blair, W. P., Raymond, J. C., Long, K. S., \& Kriss, G. A. 1995, ApJ, 454, L35

Bloemen, J. B. G. M., Strong, A. W., Mayer-Hasselwander, H. A., et al. 1986, A\&A, 154, 25

Castelletti, G., Dubner, G., Golap, K., \& Goss, W. M. 2006, A\&A, 459, 535

Dubner, G. M., \& Arnal, E. M. 1988, A\&AS, 75, 363

Dubner, G., Giacani, E., Reynoso, E., \& Parón, S. 2004, A\&A, 426, 201

Fesen, R. A., Kwitter, K. B., \& Downes, R. A. 1992, AJ, 104, 719

Ghavamian, P., Raymond, J., Hartigan, P., \& Blair, W. P. 2000, ApJ, 535, 266

Hollenbach, D. J., \& Tielens, A. G. G. M. 1999, Rev. Mod. Phys., 71, 173

Hwang, U., Flanagan, K. A., \& Petre, R. 2005, ApJ, 635, 355

Klein, R. I., McKee, C. F., \& Colella, P. 1994, ApJ, 420, 213

Koo, B.-C., Rho, J., Reach, W. T., Jung, J., \& Mangum, J. G. 2001, ApJ, 552, 175

Levenson, N. A., Graham, J. R., Aschenbach, B., et al. 1997, ApJ, 484, 304 Levenson, N. A., Graham, J. R., \& Walters, J. L. 2002, ApJ, 576, 798

Petre, R., Kriss, G. A., Winkler, P. F., \& Canizares, C. R. 1982, ApJ, 258, 22

Prasad, S. S., \& Tarafdar, S. P. 1983, ApJ, 267, 603

Reynoso, E. M., \& Dubner, G. M. 1997, A\&AS, 123, 31

Reynoso, E. M., Dubner, G. M., Goss, W. M., \& Arnal, E. M. 1995, AJ, 110, 318

Reynoso, E. M., Green, A. J., Johnston, S., et al. 2003, MNRAS, 345, 671

Sakamoto, S., Hayashi, M., Hasegawa, T., Handa, T., \& Oka, T. 1994, ApJ, 425, 641

Sasaki, M., Kothes, R., Plucinsky, P. P., Gaetz, T. J., \& Brunt, C. M. 2006, ApJ, 642, L149

Seta, M., Hasegawa, T., Dame, T. M., et al. 1998, ApJ, 505, 286

Teske, R. G., \& Petre, R. 1987, ApJ, 314, 673

Wardle, M. 1999, ApJ, 525, L101

Yamaguchi, N., Mizuno, N., Moriguchi, Y., et al. 1999, PASJ, 51, 765 